\title{
Does duration of hysteroscopy increase the risk of disease recurrence in patients with endometrial cancer? A multi-centre trial
}

\author{
CLEMENS TEMPFER ${ }^{1}$, GEORG FROESE ${ }^{2}$, BERND BUERKLE ${ }^{1}$, STEPHAN POLTERAUER $^{3}$, CHRISTOPH GRIMM $^{3}$, \\ NICOLE CONCIN $^{4}$, GERDA HOFSTETTER ${ }^{4}$, MONIKA WEIGERT ${ }^{5}$ and MARTIN K. OEHLER ${ }^{6}$ \\ ${ }^{1}$ Department of Obstetrics and Gynaecology, Ruhr University Bochum, Bochum, Germany; ${ }^{2}$ Department of Surgery, \\ St. Elisabeth Hospital, Vienna; ${ }^{3}$ Department of Obstetrics and Gynecology, Medical University of Vienna, Vienna; \\ ${ }^{4}$ Department of Obstetrics and Gynecology, Medical University of Innsbruck, Innsbruck; \\ ${ }^{5}$ Department of Obstetrics and Gynecology, Landesklinikum Wiener Neustadt, Wiener Neustadt, Austria; \\ ${ }^{6}$ Department of Gynecologic Oncology, Royal Adelaide Hospital, Adelaide, Australia
}

Received April 21, 2011; Accepted June 15, 2011

DOI: $10.3892 /$ etm.2011.309

\begin{abstract}
Women with endometrial cancer often undergo hysteroscopy during their diagnostic work-up. Whether or not the duration of hysteroscopy affects the rate of positive peritoneal cells and the duration of recurrence-free survival is unknown. In a retrospective multi-centre study, the records of 552 patients with endometrial cancer were investigated. Duration of hysteroscopy was correlated with clinicopathological parameters and patient survival data. The mean [standard deviation (SD)] duration of hysteroscopy was 18.2 (10.5) min in the study population and 17.9 (10.1) $\mathrm{min}$ and 17.9 (10.2) $\mathrm{min}$ in patients with positive $(n=109)$ and negative peritoneal cytology $(n=443)$, respectively $(p=0.9)$. There were no statistically significant correlations between duration of hysteroscopy and positive peritoneal cytology $(\mathrm{p}=0.6$; rho=-0.028), FIGO stage $(\mathrm{p}=0.2 ;$ rho $=-0.080)$, lymph node involvement $(\mathrm{p}=0.2$; rho $=0.106)$ and patient age $(\mathrm{p}=0.5$; rho $=0.033)$. Longer duration of hysteroscopy (>15 min) was not associated with positive peritoneal cytology (yes vs. no, $\mathrm{p}=0.8$ ), advanced tumour stage (FIGO I vs. II, III and IV, p=0.3), lymph node involvement (yes vs. no, $p=0.1$ ) and patient age ( $\leq 65$ vs. $>65$ years, $p=0.4$ ). In a multivariate analysis, FIGO stage $[\mathrm{p}<0.0001$; hazard ratio $(\mathrm{HR})=5.1,95 \%$ confidence interval (CI) 2.5-10.2], lymph node involvement $(\mathrm{p}=0.02 ; \mathrm{HR}=3.2,95 \%$ CI 1.2-8.8) and patient age ( $\mathrm{p}=0.003 ; \mathrm{HR}=2.4,95 \% \mathrm{CI} 1.3-4.2)$, but not duration of hysteroscopy ( $\mathrm{p}=0.4 ; \mathrm{HR}=1.2,95 \%$ CI 0.7-2.2), were associated with recurrence-free survival. We conclude that longer duration of hysteroscopy does not increase the risk of positive peri-
\end{abstract}

Correspondence to: Dr Clemens B. Tempfer, Department of Obstetrics and Gynaecology, Ruhr University Bochum, Hoelkeskampring 40, D-44625 Herne, Germany

E-mail: clemens.tempfer@marienhospital-herne.de

Key words: hysteroscopy, duration, endometrial cancer, prognosis toneal cytology and it is not an adverse prognostic factor for recurrence-free survival in patients with endometrial cancer.

\section{Introduction}

Endometrial cancer accounts for approximately $4 \%$ of all cancers in women and occurs predominantly after menopause. Some of the highest incidence rates worldwide are found in the US and European populations (1). Clinical signs and symptoms associated with endometrial cancer include postmenopausal bleeding and perimenopausal meno-metrorrhagia (2). Women presenting with these conditions undergo histological evaluation of the endometrium. Typically, endometrial tissue sampling is combined with hysteroscopy, i.e., the optical visualization of the endometrial cavity via an endoscope (2-5). Hysteroscopy is a highly accurate method and is useful in diagnosing, rather than excluding endometrial cancer in women with abnormal uterine bleeding (6). This technique, however, flushes fluid and endometrial cells into the abdomen via the fallopian tubes, thus potentially spreading malignant cells. For example, in a prospective randomized study, Nagele et al examined 30 women undergoing hysteroscopy and concomitant laparoscopy for infertility. Endometrial cells were present in the peritoneal fluid in $6 \%$ of patients before hysteroscopy and in $25 \%$ after hysteroscopy (7). A number of studies have associated hysteroscopy with an increased risk of positive peritoneal cytology in women with endometrial cancer $(8,9)$, while certain studies have raised the possibility of an adverse impact of hysteroscopy on prognosis in women with endometrial cancer (10-13).

Despite these concerns, however, hysteroscopy is considered a safe and acceptable procedure and is used extensively for the evaluation of women with postmenopausal bleeding or perimenopausal meno-metrorrhagia $(14,15)$. Whether or not the duration of hysteroscopy is a matter of concern in women with suspected endometrial cancer is yet unknown. If prolonged hysteroscopy increased the risk of positive peritoneal cytology and disease recurrence, this would have 
clinical implications regarding time restriction or avoidance of hysteroscopy in women with suspected endometrial cancer.

To investigate this issue, we performed a retrospective multi-centre study in a large series of women with endometrial cancer who underwent pre-operative hysteroscopy. The aim of our study was to investigate whether the duration of hysteroscopy is associated with positive peritoneal cytology at surgery and adverse prognosis in patients with endometrial cancer.

\section{Materials and methods}

Five hundred and fifty-two patients with endometrial cancer, treated at the Departments of Obstetrics and Gynecology of the Medical University of Vienna, Austria $(n=220)$, the Medical University of Innsbruck, Austria (n=204), the Landeskrankenhaus Klagenfurt, Austria ( $n=87)$, the Landeskrankenhaus Wiener Neustadt, Austria $(n=39)$ and the Department of Gynecologic Oncology, Royal Adelaide Hospital, Adelaide, Australia ( $n=2)$, between February 1996 and July 2009, were included in the present study. Clinical and laboratory data were extracted retrospectively from patient files.

Diagnosis of endometrial cancer was established by diagnostic hysteroscopy and dilation and curettage. Hysteroscopy was performed with saline as the distension medium. Duration of hysteroscopy was defined as the time between the start and the end of the surgical procedure. The duration of hysteroscopy was noted by the nursing staff on a surgical procedure documentation sheet. Patients were surgically staged according to the International Federation of Gynaecology and Obstetrics (FIGO)/American Joint Committee on Cancer (AJCC) classification system. Hysterectomy, bilateral salpingo-oophorectomy, cytological examination of peritoneal fluid and biopsy of any suspicious intraperitoneal or retroperitoneal lesions were performed. Pelvic and paraaortic lymphadenectomy were performed, except for tumour stages FIGO Ia and Ib (using the 1989 FIGO classification for endometrial cancer) with histological grades 1 and 2 and endometrioid histology. In patients with intermediate- or high-risk disease, adjuvant radiotherapy was provided according to standardized treatment protocols (16). A regimen of adjuvant chemotherapy using carboplatin/ paclitaxel was used in selected patients with advanced disease.

Three months after completion of primary therapy, the first follow-up visit was scheduled. Patients had follow-up visits at 3-month intervals for the following 2 years, including inspection and vaginal-rectal palpation. In the third and fourth year, visits were scheduled bi-annually and once a year from the fifth year on. When patients did not present for the scheduled follow-up visits, they were contacted by administrative personnel. In the event of any clinically suspicious symptoms and/or elevation of tumour markers, computed tomography was performed.

Values represent the means [standard deviation (SD)] in the case of a normal distribution, or the medians (range) in the case of a skewed distribution. T-tests and one-way ANOVA were used to compare the duration of hysteroscopy and clinicopathological parameters. Correlations are described by Spearman's correlation coefficient. Survival probabilities were calculated by the product limit method of Kaplan and Meier. Differences between groups were tested using the log-rank test. The results were analyzed for the endpoint of
Table I. Patient characteristics.

Parameter

Total no. of patients enrolled 552

Age at diagnosis in years, mean (SD)

$66.7(10.9)$

Histological type, n (\%)

Type I

Type II

Tumour stage, n (\%)

FIGO IA

$78(14.1)$

FIGO IB

$259(46.9)$

FIGO IC

$91(16.5)$

FIGO IIA

$24(4.3)$

FIGO IIB

$27(4.9)$

FIGO III

59 (10.8)

FIGO IV

Histological grade, n (\%)

G1

$236(42.8)$

G2

$214(38.8)$

$102(18.4)$

Peritoneal cytology, n (\%)

No. of patients with positive

$109(19.7)$

peritoneal cytology

Recurrence status, n (\%)

No. of patients with recurrent disease

Time to recurrent disease in months (SD)

Status at last observation, n (\%)

Alive with no evidence of disease

Progressive disease

$23 \quad(4.2)$

Tumour-related death

$38(6.9)$

Death due to other causes

$52(9.4)$

FIGO, International Federation of Gynaecologists and Obstetricians; $\mathrm{SD}$, standard deviation.

disease-free survival. Events were defined as recurrence, death or progression at the time of last follow-up. Survival times of disease-free patients or patients with stable disease were censored with the last follow-up date. Survival times of patients who died due to other causes were censored with the date of death. Univariate analysis and a multivariate Cox regression model for disease-free survival were performed using duration of hysteroscopy ( $\leq 15$ vs. $>15 \mathrm{~min}$ ), FIGO tumour stage (FIGO I vs. FIGO II-IV), histological grade (G1+G2 vs. G3) and histological subtype (type I vs. type II). p-values $<0.05$ were considered statistically significant. The statistical software SPSS 16.0 for Mac (SPSS 16.0.1; SPSS Inc., Chicago, IL, USA) was used for statistical analysis.

The present study was approved by the Ethics Committee of the Medical University of Vienna and the General Hospital of Vienna.

\section{Results}

Patient characteristics are provided in Table I. Lymph node status was available in 147/552 (27\%) patients. Lymph node 
Table II. Relationship between clinicopathological parameters and duration of hysteroscopy in 552 patients with endometrial cancer.

$$
\operatorname{HSC} \leq 15 \min (\mathrm{n}=312)
$$

$194(81 \%)$

$46(19 \%)$

$107(45 \%)$

$133(55 \%)$

$154(49 \%)$

$62(20 \%)$

$208^{\mathrm{b}}(67 \%)$

$249(80 \%)$

$63(20 \%)$

$297(95 \%)$

$15(5 \%)$

$11 \quad(4 \%)$

$301^{\mathrm{c}}(96 \%)$
$47(20 \%)$

$170^{\mathrm{b}}(71 \%)$

$201(84 \%)$

$39(16 \%)$

$223(93 \%)$

$17(7 \%)$

$16 \quad(7 \%)$

$224^{\mathrm{c}}(93 \%)$

Lymph node involvement

Yes

No

HSC, hysteroscopy; FIGO, International Federation of Gynaecologists and Obstetricians. ${ }^{\mathrm{a}} \mathrm{Chi}$-square test; ${ }^{\mathrm{b}}$ excluding missing values (n=65); cincluding women with and without lymph node sampling.

Table III. Univariate Kaplan Meier analysis and multivariate Cox regression model of prognostic covariates and recurrence-free survival in 552 patients with endometrial cancer.

\begin{tabular}{lccc}
\hline & Univariate & & Multivariate $^{\mathrm{b}}$ \\
\cline { 3 - 4 } & p-value & HR (95\% CI) & p-value \\
\hline FIGO stage (I vs. II-IV) & $<0.00001$ & $5.1(2.5-10.2)$ & $<0.0001$ \\
Tumour grading (G1+2 vs. G3) & 0.05000 & $1.7(0.9-3.3)$ & 0.0800 \\
Age ( $>$ 65 vs. $\leq 65$ years) & 0.00200 & $3.2(1.2-8.8)$ & 0.0030 \\
Lymph node involvement (yes vs. no) & 0.01000 & $1.2(0.8-1.8)$ & 0.0200 \\
Histological type (type I vs. II) & 0.10000 & $1.2(0.7-2.2)$ & 0.3000 \\
Duration of HSC ( $\leq 15$ vs. $>15$ min) & 0.40000 & 0.4000 \\
\hline
\end{tabular}

HR, hazard ratio; 95\% CI, 95\% confidence interval; HSC, hysteroscopy. ${ }^{\mathrm{a}}$ Log-rank test; ${ }^{\mathrm{b}}$ multivariate Cox regression analysis.

involvement was noted in $27 / 552(5 \%)$ patients. Adjuvant radiotherapy was administered to $298 / 552(54 \%)$ patients and 45/552 (8\%) patients received adjuvant chemotherapy.

The mean (SD) duration of hysteroscopy in all patients was 18.2 (10.5) min. The mean (SD) duration of hysteroscopy in patients with positive $(n=109)$ and negative peritoneal cytology $(\mathrm{n}=443)$ was not statistically different [17.9 (10.1) min vs. 17.9 (10.2) min, respectively; $\mathrm{p}=0.9$ ]. There were no statistically significant correlations between duration of hysteroscopy (as a continuous variable) and positive peritoneal cytology $(\mathrm{p}=0.6 ;$ rho=-0.028), FIGO stage $(\mathrm{p}=0.2 ; \mathrm{rho}=-0.080)$, lymph node involvement $(\mathrm{p}=0.2$; rho $=0.106)$ and patient age $(\mathrm{p}=0.5$; rho=0.033). The associations between duration of hysteroscopy and clinicopathological parameters are provided in Table II. Longer duration of hysteroscopy was not associated with positive peritoneal cytology, advanced tumour stage, histological grade, histological type, lymph node involvement or advanced patient age.

In a univariate analysis, tumour stage (FIGO I vs. II-IV), histological grade (G1+G2 vs. G3), histological type (type I vs. II), lymph node involvement (yes vs. no) and patient age (as a continuous variable), but not duration of hysteroscopy (as a continuous variable), were associated with disease-free survival. In a multivariate analysis, tumour stage, histological grade, histological subtype and lymph node involvement were associated with disease-free survival. Results of the univariate 


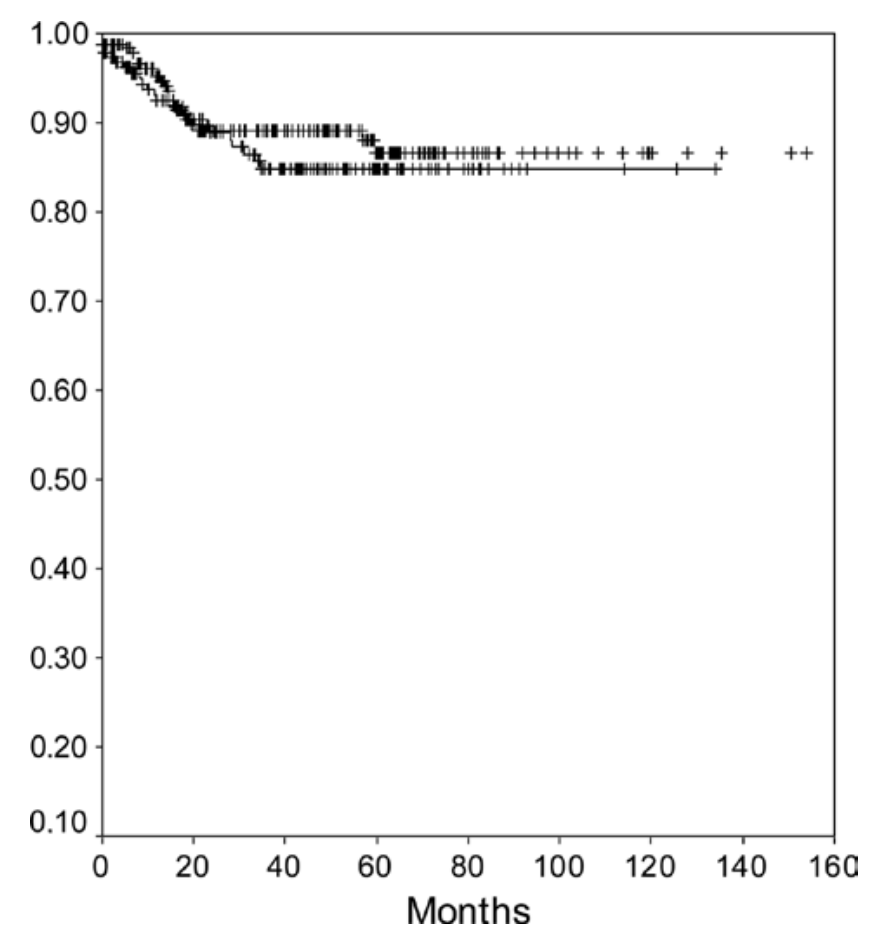

Figure 1. Recurrence-free survival analysis of 552 women with endometrial cancer according to duration of hysteroscopy $\leq 15 \mathrm{~min}$ (upper line) and $>15$ min (lower line).

and multivariate Cox-regression models and log-rank tests with respect to disease-free survival are shown in Table III. When patients were grouped according to the duration of hysteroscopy, patients with a duration of hysteroscopy $\leq 15$ and $>15 \mathrm{~min}$ had a 5-year recurrence-free survival rate of 87 and $85 \%$, respectively $[\mathrm{p}=0.4$; hazard ratio $(\mathrm{HR})=1.2,95 \%$ confidence interval (CI) 0.7-2.2] (Fig. 1).

\section{Discussion}

In the present study, we investigated whether a prolonged duration of hysteroscopy leads to a higher risk of positive peritoneal cytology and an increased risk of recurrence in women with endometrial cancer. We found that the duration of hysteroscopy was not associated with positive peritoneal cytology nor was it a prognostic parameter for recurrence-free survival. We conclude from these data that longer duration of hysteroscopy in patients with endometrial cancer is oncologically safe. It does not increase the risk of positive peritoneal cytology and does not adversely impact prognosis.

A number of studies have demonstrated that hysteroscopy increases the risk of positive peritoneal cytology and upstaging among women with endometrial cancer. For example, in a recent meta-analysis of 9 studies and 1,015 patients, Polyzos et al reported a significantly higher rate of malignant peritoneal cytology after hysteroscopy with an odds ratio of 1.78 (9). Thus, even though there is no clear evidence of an adverse prognostic effect of hysteroscopy and positive peritoneal cytology is no longer part of the FIGO staging system (9-13), concerns remain. Since endometrial cancer in general is associated with a favorable prognosis, subtle effects may be difficult to detect. Also, relevant confounders, such as the histological type of endometrial cancer or the duration of hysteroscopy, may influ- ence the effect of this procedure on cancer cell spread and prognosis. These issues are of clinical relevance, since the duration of hysteroscopy is a potential risk factor under the control of the treating physician. Therefore, we investigated the influence of the duration of hysteroscopy on the risk of positive peritoneal cytology and recurrence-free survival in a large retrospective cohort study using a multi-centre study approach. We found that duration of hysteroscopy, both as a continuous variable and as a dichotomous variable with an arbitrary cutoff point of $15 \mathrm{~min}$, was not associated with the rate of positive peritoneal cytology at the time of surgery or with the duration of disease-free survival. The results of our study have clinical implications, since they demonstrate that hysteroscopy can be used safely in women with suspected endometrial cancer even when a prolonged procedure is necessary to obtain an optimal diagnostic result. Also, these data support the new 2009 FIGO staging revision which omits stage IIIA based on positive peritoneal cytology only.

Our study has limitations which include the retrospective study design and the inclusion of multiple surgeons and centers. Thus, we cannot exclude selection and ascertainment bias. For example, technical expertise and equipment standards may vary among surgeons and centers as well as over time, and specific patient populations may or may not preferentially choose the centers included in this study. Also, hydrostatic pressure during hysteroscopy was not assessed. On the other hand, hysteroscopy is a relatively simple and well-standardized procedure, thus minimizing these effects. The strength of our study is the large number of patients allowing for an accurate estimate of the association between duration of hysteroscopy and clinicopathological parameters and prognosis.

In summary, our results demonstrate that prolonged duration of hysteroscopy is not associated with an increased risk of positive peritoneal cytology and it does not adversely affect the extent of recurrence-free survival. Therefore, hysteroscopy may be used safely in women with suspected endometrial cancer, even when the procedure requires an extended duration due to technical or other circumstances.

\section{References}

1. Parkin DM, Whelan SL, Ferlay J, Teppo L and Thomas DB: Cancer Incidence in Five Continents. Vol. VIII. IARC Press, Lyon, 2002.

2. Creasman WT, Odicino F, Maisonneuve P, Beller U, Benedet JL, Heintz AP, Ngan HY, Sideri M and Pecorelli S: Carcinoma of the corpus uteri. J Epid Biostat 6: 47-86, 2001.

3. Coulter A, Kelland A and Long A: The management of menorrhagia. Effective Health Care Bull 9: 1-14, 1995.

4. Spencer CP and Whitehead MI: Endometrial assessment revisited. Br J Obstet Gynaecol 106: 623-632, 1999.

5. Mencaglia L: Hysteroscopy and adenocarcinoma. Obstet Gynecol Clin North Am 22: 573-579, 1995.

6. Clark TJ, Voit D, Gupta JK, Hyde C, Song F and Khan KS: Accuracy of hysteroscopy in the diagnosis of endometrial cancer and hyperplasia: a systemic quantitative review. JAMA 288: 1610-1621, 2002.

7. Nagele F, Wieser F, Deery A, Hart R and Magos A: Endometrial cell dissemination at diagnostic hysteroscopy: a prospective randomized cross-over comparison of normal saline and carbon dioxide uterine distension. Hum Reprod 14: 2739-2742, 1999.

8. Obermair A, Geramou M and Gucer F: Does hysteroscopy facilitate tumor cell dissemination? Incidence of peritoneal cytology from patients with early stage endometrial carcinoma following dilatation and curettage versus hysteroscopy and dilatation and curettage. Cancer 88: 139-143, 2000. 
9. Polyzos NP, Mauri D, Tsioras S, Messini CI, Valachis A and Messinis IE: Intraperitoneal dissemination of endometrial cancer cells after hysteroscopy: a systematic review and meta-analysis. Int J Gynecol Cancer 20: 261-267, 2010

10. Obermair A, Geramou M, Tripcony L, Nicklin JL, Perrin L and Crandon AJ: Peritoneal cytology: impact on disease-free survival in clinical stage I endometrioid adenocarcinoma of the uterus. Cancer Lett 164: 105-110, 2001.

11. Selvaggi L, Cormio G, Ceci O, Loverro G, Cazzolla A and Bettocchi S: Hysteroscopy does not increase the risk of microscopic extrauterine spread in endometrial carcinoma. Int J Gynecol Cancer 13: 223-227, 2003.

12. Cicinelli E, Tinelli R, Colafiglio G, Fortunato F, Fusco A Mastrolia S, Fucci AR and Lepera A: Risk of long-term pelvic recurrences after fluid minihysteroscopy in women with endometrial carcinoma: a controlled randomized study. Menopause 17 511-515, 2010.
13. Ben-Arie A, Tamir S, Dubnik S, Gemer O, Ben Shushan A, Dgani R, Peer G, Barnett-Griness O and Lavie O: Does hysteroscopy affect prognosis in apparent early-stage endometrial cancer? Int J Gynecol Cancer 18: 813-819, 2008

14. Serden SP: Diagnostic hysteroscopy to evaluate the cause of abnormal uterine bleeding. Obstet Gynecol Clin North Am 27: 277-286, 2000.

15. O'Rogerson L and Duffy S: A national survey of outpatient hysteroscopy. Gynecol Endosc 10: 343-348, 2001.

16. Nag S, Erickson B, Parikh S, Gupta N, Varia M and Glasgow G: The American Brachytherapy Society recommendations for high-dose-rate brachytherapy for carcinoma of the endometrium. Int J Radiat Oncol Biol Phys 48: 779-790, 2000. 